\title{
DEVELOPMENT OF A NEW RECURSIVE MEDIAN FILTERING SCHEME FOR PROCESSING POTENTIOMETER SIGNAL IN CSRDM OF PFBR
}

\author{
M. Mohana*, Sudheer Patri, S. Narmadha, B. Babu, V. Prakash, K. K. \\ Rajan \\ Indira Gandhi Centre for Atomic Research \\ Kalpakkam, Tamil Nadu, India \\ *manomohana@igcar.gov.in
}

\begin{abstract}
Prototype Fast Breeder Reactor (PFBR) which is in advanced stage of construction at Kalpakkam has two shutdown systems, namely - Control \& Safety Rod Drive Mechanisms (CSRDM) and Diverse Safety Rod Drive Mechanisms (DSRDM). Since response time of Electromagnet (EM) present in CSRDM is an important safety parameter, it is measured and monitored periodically. In PFBR, measurement of response time of EM is done by Current Decay method. An alternate approach to measure EM response time is Displacement Method, wherein it is proposed to utilise the existing potentiometer in CSRDM for position monitoring to measure EM response time. In light of this, a study was carried out to probe the feasibility of its implementation. To aid in the study, a new Recursive Median filtering scheme termed as 'Multipass recursive median filter with variable window' was developed to process the unconditioned potentiometer signal in Displacement method. EM response time obtained from Displacement Method and that obtained through Current decay method were found to be in good agreement with each other. This paper details the new Recursive Median filtering scheme developed.
\end{abstract}

\section{KEY WORDS}

CSRDM, EM response time, Multi-pass recursive median filter with varying window length, Coefficient of variation.

\section{INTRODUCTION}

PFBR is a 500 MWe, sodium cooled, pool type, mixed oxide (MOX) fuelled Fast Breeder Reactor under construction at Kalpakkam in India. It has two independent, diverse, fast acting and fail safe shut down systems to achieve the required level of safety and reliability [1]. The absorber rod of the first system is called Control \& Safety Rod (CSR) and of the other system is called Diverse Safety Rod (DSR). Their respective drive mechanisms are called CSRDM and DSRDM. Enriched boron carbide $\left(\mathrm{B}_{4} \mathrm{C}\right)$ is used as the absorber material. The CSR system consists of nine rods and their associated mechanisms. The DSR system consists of three rods and their associated mechanisms. The main functions of CSRDM are to facilitate start-up, control of reactor power, controlled shutdown of reactor by raising and lowering of CSR, and SCRAM the reactor under abnormal conditions. While the only function of DSRSM is to SCRAM the reactor under abnormal conditions. SCRAM is achieved by rapid insertion of Control \& Diverse Safety Rods

Natarajan Meghanathan, et al. (Eds): SIPM, FCST, ITCA, WSE, ACSIT, CS \& IT 06, pp. 109-118, 2012. (C) CS \& IT-CSCP 2012 
into the core. SCRAM is acronym for Safety Control Rod Accelerated Movement. Schematic view of CSRDM is shown in Figure 1.

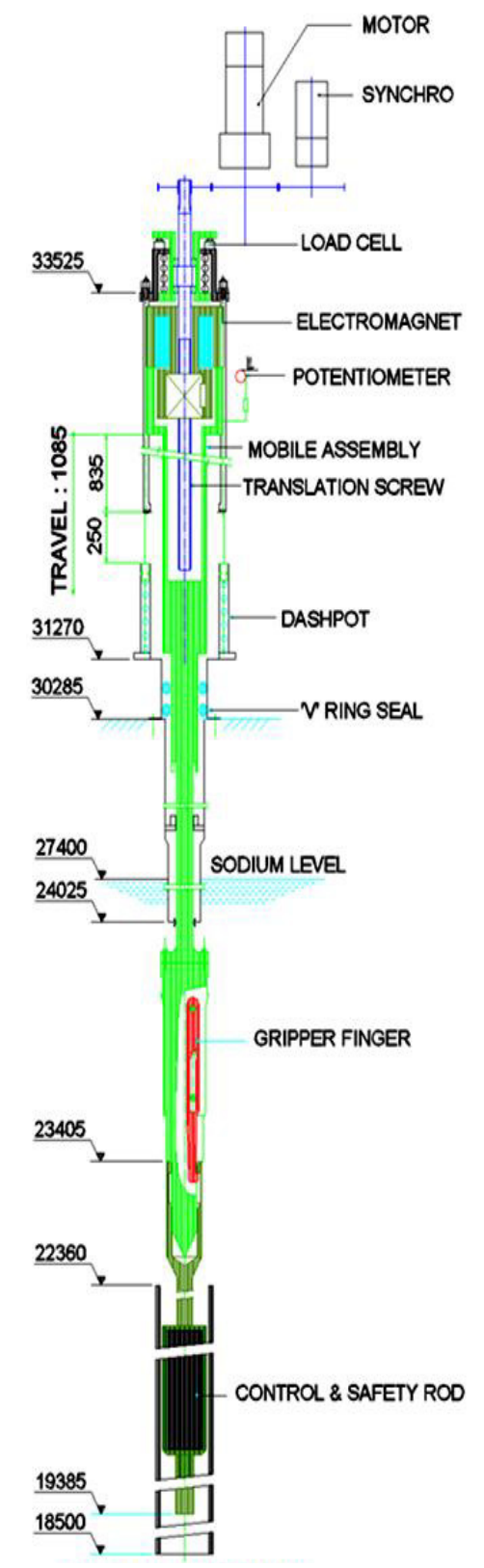

Figure 1. Schematic view of CSRDM

In CSRDM, an EM acts as the SCRAM release device. Schematic view of the EM is shown in Figure 2. Free-wheeling diode circuit shown in Figure 3 is used to dissipate the stored energy in the electromagnet when its power supply is interrupted. During normal operation, Mobile Assembly (MA) of CSRDM is held by an EM located at the top of CSRDM. On receiving the SCRAM signal, EM is de-energised and the MA of CSRDM along with CSR is released to fall under gravity [2]. But a finite delay is observed in the start of movement of CSR even after 
receiving the SCRAM signal which is due to EM response time and it depends on $\mathrm{L} \& \mathrm{R}$ values of the coil and its associated decay circuit. During SCRAM action, reactivity of the core decreases only after CSR starts moving down into the core. Thus, EM response time is an important safety parameter, with maximum allowable value of $100 \mathrm{~ms}$. Response time of an EM is defined as the time interval between the interruption of power supply to EM and the travel of MA by $1 \mathrm{~mm}$ after detachment [3]. EM response time measurement is done in two ways, namely- Current Decay Method and Displacement Method.

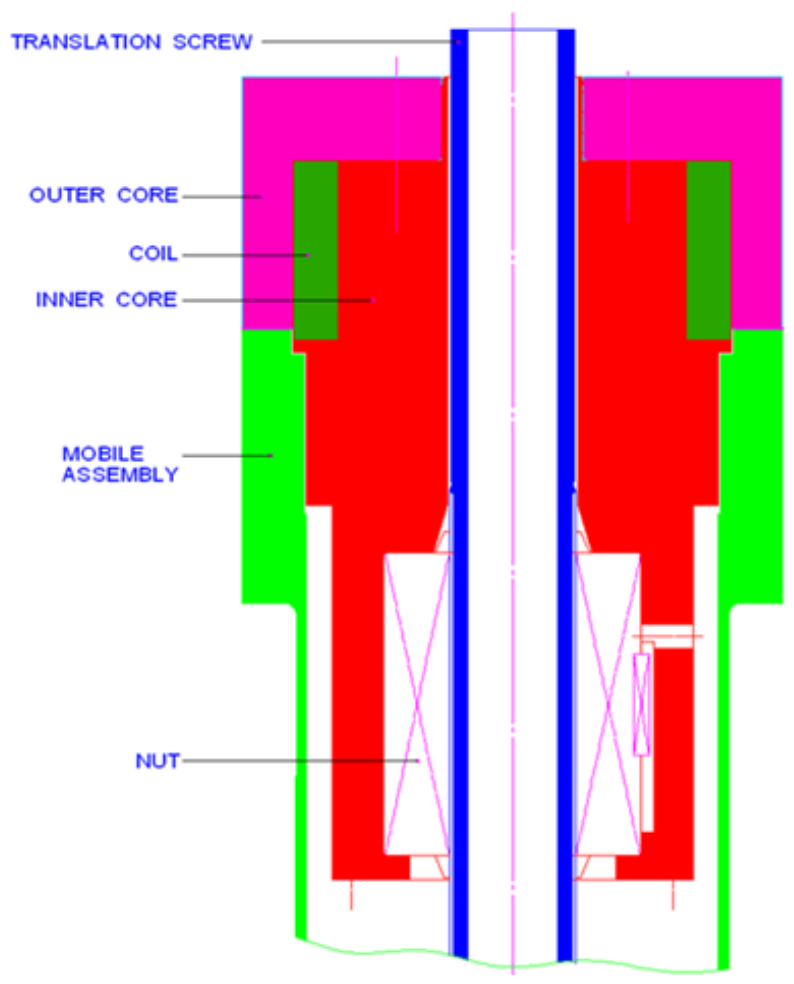

Figure 2. Electromagnet of CSRDM

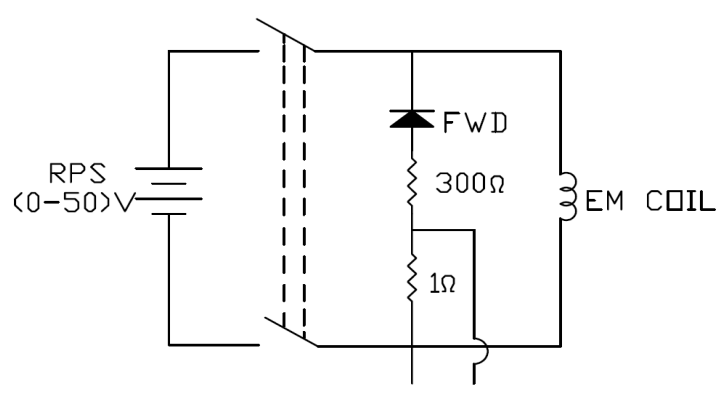

Figure 3. Energising and de-energising circuit of EM

\section{Current Decay Method}

Generally, EM response time is measured by Current Decay method. Voltage drop across $1 \Omega$ resistor in series with free-wheeling diode of circuit shown in Figure 3 is used for indirectly measuring the current through the circuit. A typical EM response time curve is shown in Figure 4. When the EM is in energised condition, current does not flow through the free-wheeling diode, 
and hence voltage across $1 \Omega$ resistor is zero. But, immediately after the EM is de-energised, the decay current starts to flow through free-wheeling diode and hence through $1 \Omega$ resistor also, which results in first peak in the EM response time curve. When the current decays to a value below the minimum holding current of EM, the MA starts separating from EM. Beyond this instant, current through EM increases, since the tendency of a magnetic circuit is to oppose any change in flux linked with the coil (in this case - decrease in flux due to the separation of the MA). Once the MA is separated far enough from EM, current through EM starts decreasing again. This results in second peak. The time interval between the first and second peaks is the EM response time, as indicated in Figure 4.

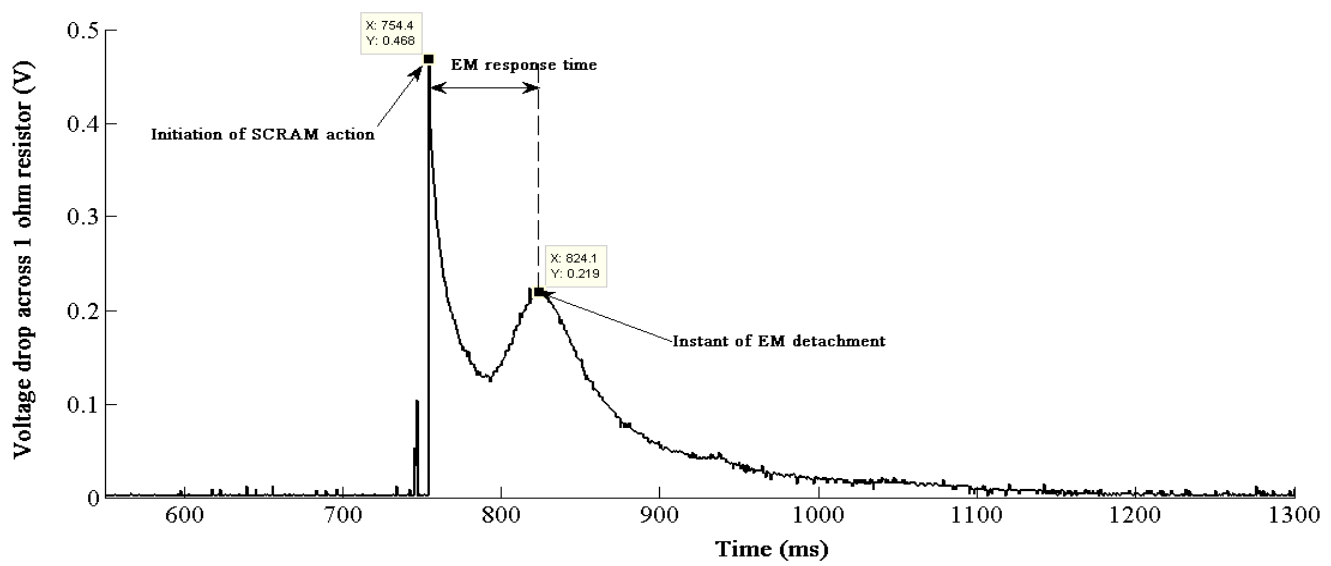

Figure 4. A typical EM response curve (taken for a drop height of $1085 \mathrm{~mm}$ )

\section{DisPlacement MethoD}

Since EM response time is an important safety parameter, implementing redundancy in its measurement ensures increased safety of the system. In light of this an alternate approach to measure EM response time termed as Displacement method was proposed. A draw wire type potentiometer is in use in CSRDM to continuously monitor the position of MA. As an extended scope, this potentiometer can be utilised for EM response time measurement by analysing its output signal acquired during SCRAM action. EM response time is defined as the time interval between the instant of initiation of SCRAM action and the instant of completion of $1 \mathrm{~mm}$ travel of MA. In this technique, the time instant of completion of $1 \mathrm{~mm}$ travel alone is obtained from potentiometer output. While the time instant of initiation of SCRAM action is taken directly from the corresponding SCRAM signal [4]. 


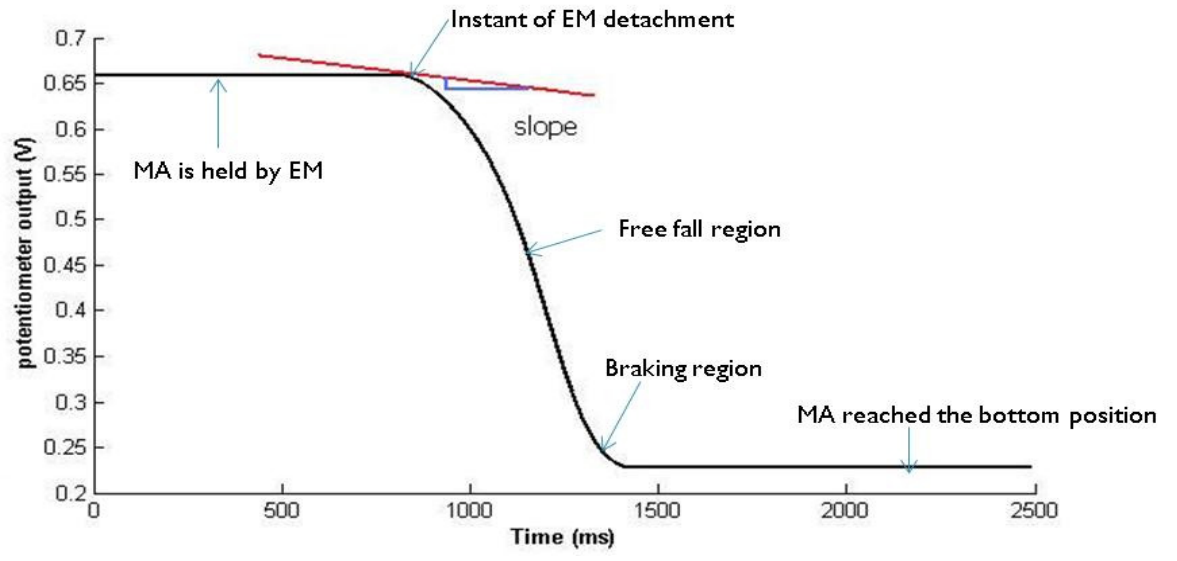

Figure 5. Ideal Displacement vs. time curve during SCRAM

An ideal Displacement vs. time curve obtained using potentiometer signal during SCRAM action is shown in Figure 5. Displacement is expressed in terms of potentiometer output. Initially the curve is flat because the MA is held stationary by the EM. When the EM is de-energised MA starts moving, but with some time delay due to EM response time. MA moves with uniform acceleration till the end of free fall and the corresponding portion of the curve is parabolic. During braking travel MA is subjected to deceleration. Thus the exact time instant of EM detachment can be obtained by finding the time instant at which the slope of the curve turns negative from zero.

An ideal SCRAM signal obtained from the SCRAM push button is shown in Figure 6. The curve is flat at a maximum value until SCRAM is initiated, and after initiation of SCRAM the curve is flat again but at a minimum value. Hence instant of initiation of SCRAM is the instant at which this change of state occurs.

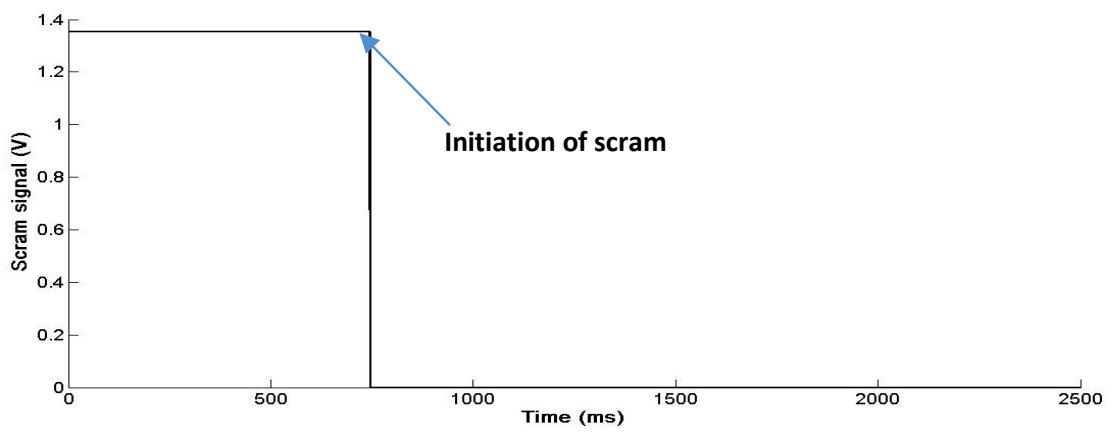

Figure 6. An ideal SCRAM signal

\section{NEED FOR SIGNAL PROCESSING}

Displacement vs. time curve obtained from the potentiometer is not conditioned as shown in Figure 5, but is mixed with random noise as shown in Figure 7. It can be seen that the signal exhibits small random variations around the actual value with spikes at few instances. These spikes are due to the noise pick-up during actuation of various micro-switches present in the mechanism, namely- SCRAM switch, $250 \mathrm{~mm}$ position switch, $260 \mathrm{~mm}$ position switch, etc. Presence of noise makes it difficult to determine slope of the curve. Hence the potentiometer signal should be subjected to signal processing to reduce the noise and also to tone up the signal and make it suitable for extraction of information regarding instant of EM detachment. There are 
many kinds of filters, each with distinct advantages for a particular application. Median filter was chosen for this application because it can remove the spike noise and smooth the signal, while preserving the useful details of the signal.

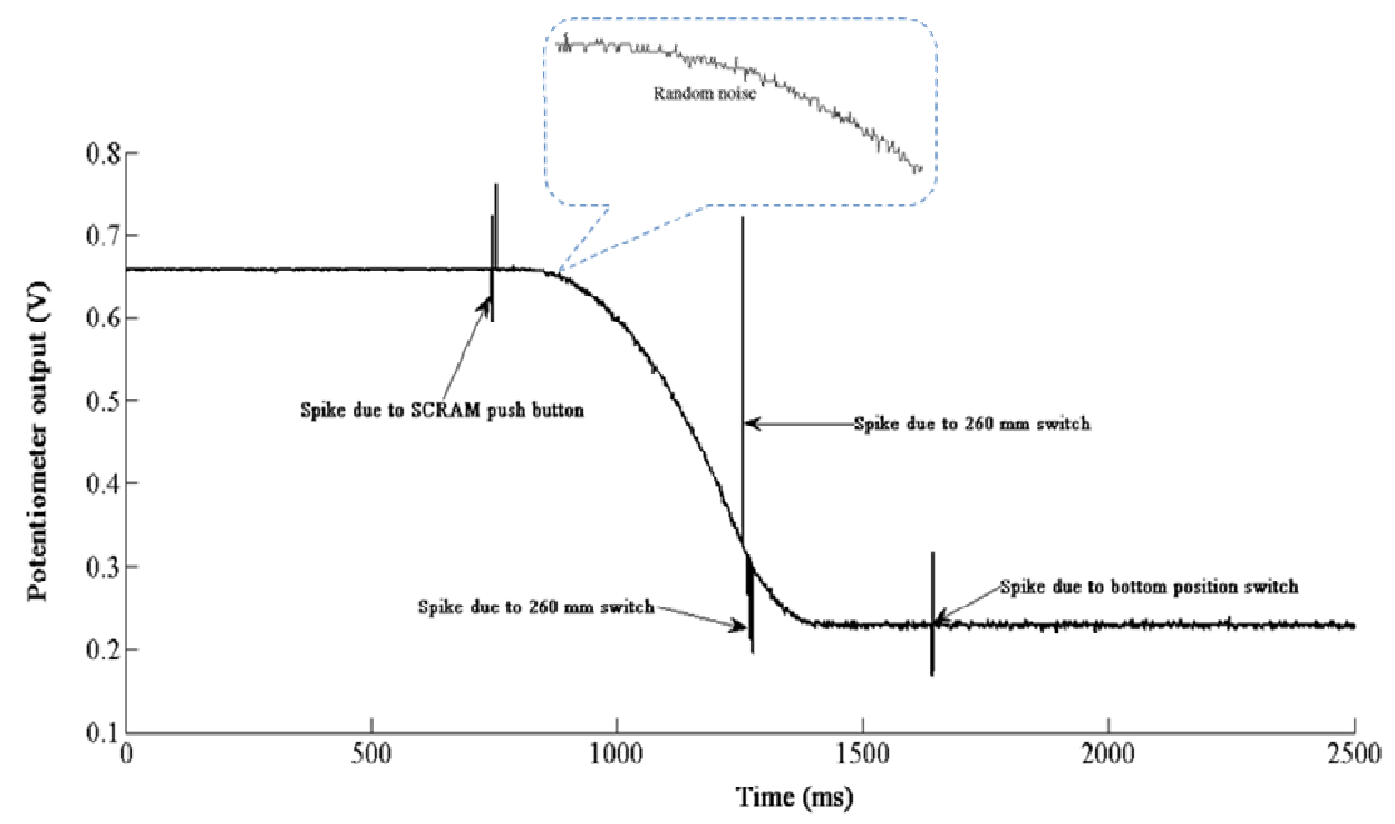

Figure 7. A typical Displacement vs. time curve

\section{NEW RECURSIVE MEDIAN FILTER}

The robustness of median against the outliers has enabled its applicability as filters in signal and image processing. The running median was first suggested as a nonlinear filter by Tukey in 1974 [5]. Accordingly, if a discrete signal $\mathbf{x}[\mathbf{n}]$ is passed through a 1-D median filter with window length of $w=2 k+1$, the filtered output is given by

$$
\mathrm{y}[\mathrm{n}]=\operatorname{median}(\mathrm{x}[\mathrm{n}-\mathrm{k}], \ldots, \mathrm{x}[\mathrm{n}], \ldots, \mathrm{x}[\mathrm{n}+\mathrm{k}])
$$

This direct technique is most often referred as Standard Median (SM) filter or non-recursive median filter.

To be able to filter the outmost input samples, where parts of the window fall outside the input signal, the input signal is appended to the required size. The appending of the input signal is commonly performed by replicating the outmost input samples as many times as needed. This appending strategy is referred to as the first and last values carry-on appending strategy [6], and is used throughout the paper.

Repeated application of the median filter on a signal of finite length ultimately results in a sequence, termed as root signal, which is invariant to further additional passes of the median filter [7]. This process of repeated application of a filter is termed as Multi-pass filtering.

A simple extension of Standard Median Filter is Recursive Median Filter (RMF). Here, the point $\mathrm{x}[\mathrm{n}]$ is replaced with the output of median filter $\mathrm{y}[\mathrm{n}]$ before shifting the window to the next position, [6]. Its output is given by 


$$
y[n]=\operatorname{median}(y[n-k], \ldots, y[n-1], x[n], x[n+1], \ldots, x[n+k])
$$

Recursive median filters have stronger noise attenuation capability than their non-recursive counter parts and a fast convergence of signals to roots. In fact a $1 \mathrm{D}$ signal will be reduced to a root after one pass of a Recursive median filter [7].

In fixed length median filters such as Standard Median Filter and Recursive Median Filter, window length is chosen such that, it is at least twice the width of the impulsive (or spike) noise. If window length is less than the optimum value, the filtered signal will not be completely free of noise. If window length is more than the optimum value, noise suppression will be more, but at the same time useful details also get lost along with the noise. Hence selection of an optimum window length is important in fixed length median filters. To overcome this problem, Lin and Wilson proposed adaptive median filters [8].

In this paper a new recursive median filtering scheme termed as 'Multi-pass Recursive median filter with variable window length' was developed to improve the potentiometer signal and make it suitable for measuring EM response time. As the name suggests, recursive median filtering is applied repeatedly by the proposed filter. Accordingly, we start the first iteration of recursive median filtering with smallest window length (3) and keep on increasing the window length of the

filter in further iterations. Repeated application of the filter is done until we get a root signal. The root signal thus obtained is the filtered output of the unconditioned potentiometer signal. As the window length is automatically decided, the new recursive median filter eliminates the problems faced with selection of optimum window length. Moreover, automatic determination of optimum window size facilitates the online implementation of the filter without user interference. Output of the proposed filter at a particular iteration ' $\mathrm{i}$ ' with window length $w_{i}=w_{i-1}+a$ is given by

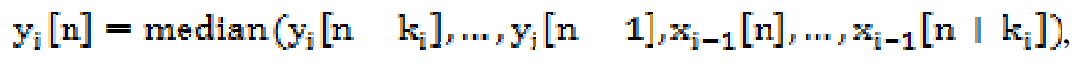

Where, $k_{\mathrm{i}}=\frac{w_{\mathrm{i}}-1}{2}$.

Algorithm for the proposed filter can be summarised as follows:

a. Obtain the input signal, $x[n]$.

b. Define a variable 'i' to count the number of iteration / number of times the filter has been applied repetitively.

c. Define a variable ' $w_{i}$ ' for the length of the window and set its initial value to be 3 .

d. Define a constant ' $a$ ' which determines the step by which length of the window should be increased at each iteration.

e. Apply Recursive median filter with window of length $w_{i}$ to the input signal $x[n]$ and obtain filtered signal $\mathrm{y}_{\mathrm{i}}[\mathrm{n}]$.

f. Compare the filtered signal $\mathrm{y}_{\mathrm{i}}[\mathrm{n}]$ with the input signal $\mathrm{x}[\mathrm{n}]$.

i. If they are same, jump to step $\mathrm{j}$

ii. Else, proceed to next step

g. Increase the length of window by ' $a$ '. So the present window length becomes $\mathrm{w}_{\mathrm{i}}=\mathrm{w}_{\mathrm{i}-1}+\mathrm{a}$.

h. Apply Recursive Median filter with window of length $w_{i}$ to the previous output of the filter $\mathrm{y}_{\mathrm{i}-1}[\mathrm{n}]$ to get a filtered signal $\mathrm{y}_{\mathrm{i}}[\mathrm{n}]$.

i. Compare the present and previous outputs of the filter, i.e., $y_{i}[n]$ and $y_{i-1}[n]$.

i. If they are same, jump to step $j$

ii. Else, repeat steps (g) to (i).

j. The present output of the filter ' $\mathrm{y}_{\mathrm{i}}[\mathrm{n}]$ ' is the de-noised root signal of potentiometer output that can be used for further analysis in the study. 
Software version of this algorithm was created in MATLAB.

\section{RESULTS AND DISCUSSION}

\subsection{Performance analysis of the new Recursive median filtering scheme}

Performance of the filters depends on how well they can suppress undesired parts of the signal and how well desired information is retained. Performance analysis of the new recursive median filter is done in terms of Coefficient of variation $(\mathrm{CoV})$. $\mathrm{CoV}$ is defined as the standard deviation $(\sigma)$ divided by mean $(\mu)$, multiplied by 100 percent [9].

$$
\operatorname{coV}=\frac{\sigma}{\mu} \times 100(\%)
$$

It is also referred to as Relative Standard Deviation (RSD) and is identical to the inverse of Signal-to-Noise Ratio (SNR). Better data possess a higher value for the SNR and a lower value for the CoV. Performance of the new recursive median filter was compared with other variants of median filters such as Standard Median filter, Multi-pass non-recursive median filter and Recursive median filter. Potentiometer data obtained during various SCRAM tests were utilised for this purpose. Figure 8 compares the $\mathrm{CoV}$ values of 6 different data filtered using the above mentioned techniques.

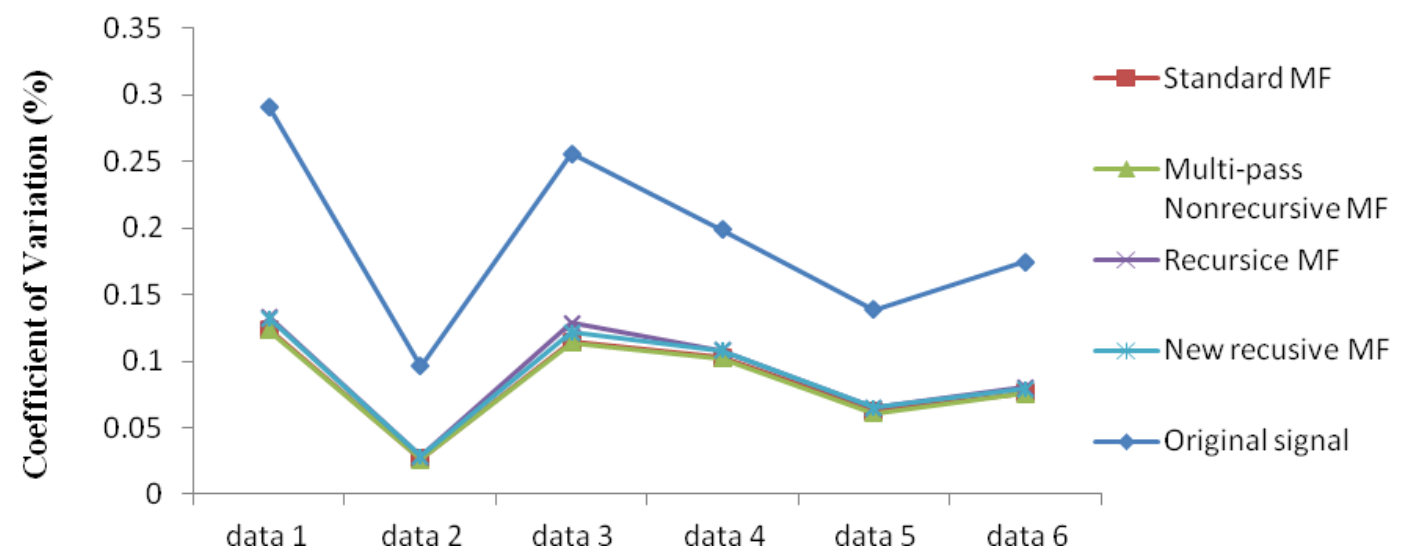

Figure 8. Performance comparison of new recursive median filtering scheme with other variants of median filters

It can be observed in Figure 8 that the original potentiometer signal which is unconditioned has a high $\mathrm{CoV}$ value indicating the presence of noise. While, all median filtered signals have low $\mathrm{CoV}$ values, indicating the absence of noise. Performance of the new recursive median filtering scheme is observed to be close to the other variants of median filters. It is advantageous over Standard Median Filter, Recursive Median Filter and multi-pass non-recursive median filter as determination of optimum window size is done by the algorithm itself, whereas in other techniques it has to be decided by the user based on the impulse width. Though numerous adaptive median filtering algorithms exist to overcome the problem of selection of optimum window size, this new recursive median filtering scheme is found to be less complex than them. Hence this technique was adopted to eliminate the noise present in the potentiometer signal of displacement method. A typical displacement vs. time curve obtained from the potentiometer signal filtered using the new recursive median filter is shown in Figure 9. 


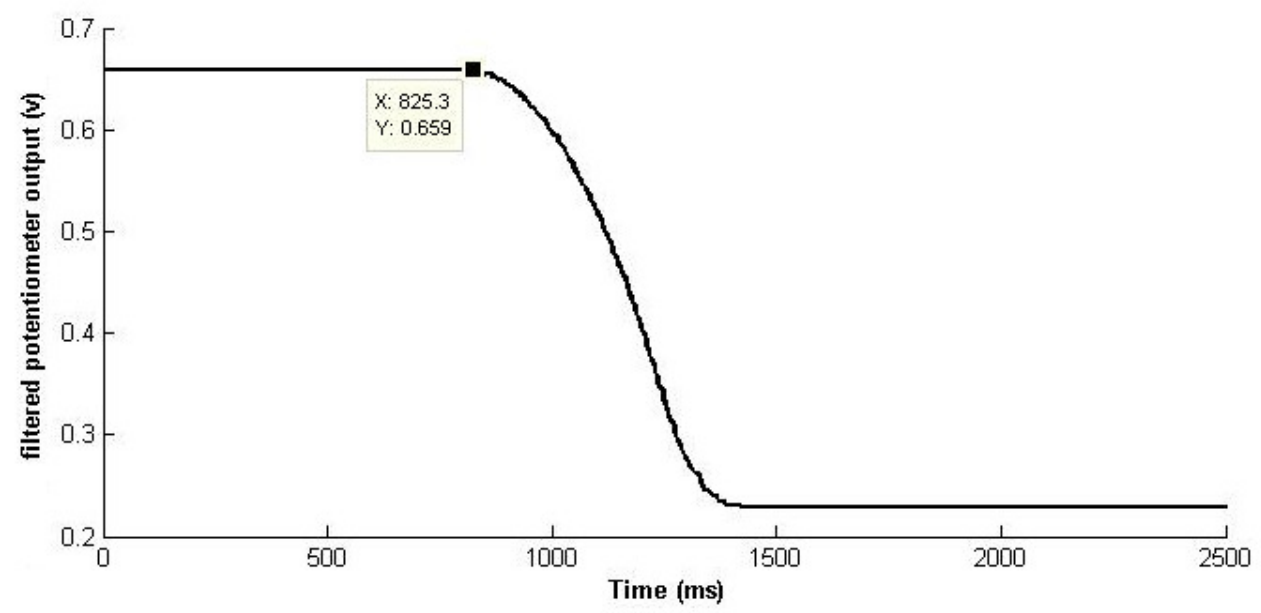

Figure 9. Displacement vs. time curve from potentiometer signal filtered by proposed filter

\subsection{Feasibility study of Displacement method}

In order to demonstrate the feasibility of Displacement method, data obtained during various SCRAM test of CSRDM were analysed. Accordingly the potentiometer signals filtered using the new recursive median filter was used to calculate EM response time through displacement method. The same was calculated using Current Decay Method also. Figure 10 compares the results of Current Decay Method and Displacement Method on various SCRAM test data. It can be observed from Figure 10 that the results of current decay method and displacement method are in good agreement with each other.

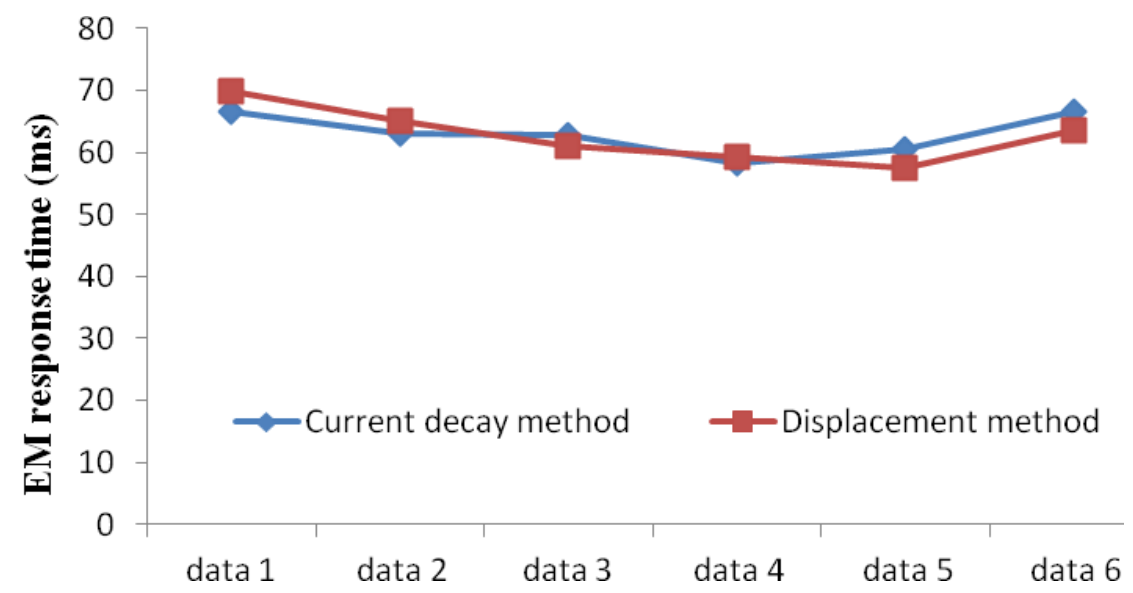

Figure 10. Comparison of results of displacement method with current decay method

\section{CONCLUSION}

The EM response time forms an important safety parameter. In PFBR response time of EM in CSRDM is measured using Current Decay Method. A feasibility study on implementing Displacement method to measure EM response time was carried out. For this purpose a new Recursive Median filtering scheme was developed to filter the noise present in the potentiometer signal. The performance of the filter was found to be close to that of other variants of the median filter. Also it was found to be advantageous over its other variants in terms of selection of 
optimum window size and complexity. Hence it was used in the feasibility study to filter the potentiometer signals to be used in displacement method. EM response time obtained from Displacement method was compared with that obtained through Current decay method and they are in good agreement with each other.

\section{REFERENCES}

[1] S. C. Chetal et.al, April 2006, "The design of the Prototype Fast Breeder Reactor" Nuclear Engineering and Design, Volume 236, Issues 7-8, Pages 852-860

[2] V. Rajan babu, "Description of Control and Safety Rod Drive Mechanism", PFBR/31430/DN/1025/R-B

[3] S. Sundarasekaran and R. Prabhakar, "Testing of PFBR CRDM electromagnet - Interim report", IGC/EDD/99221/EX/3000/R-0

[4] M. Mohana et.al, "Electromagnet response time measurement using potentiometer output in CSRDM - Feasibility study", FRTG/99220/EX/3013/R-A

[5] J.W. Tukey, 1974, "Nonlinear (nonsuperposable) methods for smoothing data", Proc. Congr. Rec. EASCOM 74, Pages 673-681.

[6] Moncef Gabbouj et.al, 1992, "Convergence behavior and root signal sets of stack filters," Circuits, Syst. Signal Process., Special Issue Median and Morphological Filtering, vol. 11, no. 1, pp. 171-194.

[7] T. A. Nodes and N. C. Gallagher, 1982, "Median filters: Some modifications and their properties," IEEE Trans. Acoust., Speech, Signal Processing, vol. ASSP-30, pp. 739-746.

[8] H. M. Lin and A. N. Wilson, Jr., June 1988, "Median filters with adaptive length," IEEE Trans. Circuits Syst., vol. 35 , no. 6 .

[9] Steven W. Smith, 1997, “The Scientist and Engineer's Guide to Digital Signal Processing”, California Technical Pub. 